\title{
Increased LEF1 Expression and Decreased Notch2 Expression Are Strong Predictors of Poor Outcomes in Colorectal Cancer Patients
}

\author{
Wen-Juan Wang, Yu Yao, Li-Li Jiang, Ting-Hua Hu, Jie-Qun Ma, \\ Zhi-Ping Ruan, Tao Tian, Hui Guo, Shu-Hong Wang, and Ke-Jun Nan \\ Department of Oncology, First Affiliated Hospital of Medical College of Xian Jiaotong University, Xian, Shaanxi 710061, China
}

Correspondence should be addressed to Shu-Hong Wang; wsh2003@126.com and Ke-Jun Nan; nankejun@126.com

Received 23 April 2013; Revised 14 July 2013; Accepted 5 August 2013

Academic Editor: Ahmed O. Kaseb

Copyright (C) 2013 Wen-Juan Wang et al. This is an open access article distributed under the Creative Commons Attribution License, which permits unrestricted use, distribution, and reproduction in any medium, provided the original work is properly cited.

\begin{abstract}
Background/Objective. We aimed to examine the expression of lymphoid enhancer factor 1 (LEF1) and Notch2 in colorectal cancer (CRC) and their association with clinicopathologic variables and CRC patients' prognosis. Methods. Immunohistochemistry, quantitative real-time polymerase chain reaction (qRT-PCR), and Western blot analysis were performed to assess the expression of LEF1 and Notch2 in 184 patients with CRC. Results. We observed a strong negative correlation between LEF1 expression and Notch2 expression $(P<0.001)$. Both LEF1 mRNA and protein expression increased while the Notch 2 mRNA and protein expression decreased in tumor specimens compared with the matched paratumorous normal tissue $(P<0.001)$. An increase in LEF1 protein expression was significantly associated with lymph node metastases, distant metastasis, advanced TNM (tumor-node-metastasis) stage, and shorter overall survival. A decrease in Notch2 protein expression was associated with poorly differentiated tumors, lymph node metastases, distant metastasis, advanced TNM stage, and shorter overall survival of patients. In the multivariate Cox regression analysis, the LEF1 protein expression $(P<0.001)$, Notch2 protein expression $(P<0.001)$, TNM stage $(P<0.001)$, and the combination of increased LEF1 protein coexpression and decreased Notch2 protein coexpression $(P<0.001)$ were found to be independent prognostic indicators in CRC. Conclusion. Our results suggest that increased LEF1 coexpression and decreased Notch2 coexpression represent a risk factor for poor overall survival of CRC patients.
\end{abstract}

\section{Introduction}

Over the last few decades, a significant decline in cancerrelated mortality of colorectal cancer (CRC) has been observed due to the considerable progress in the diagnosis and treatment, but CRC still remains a major public health problem throughout the world. Colorectal cancer is ranked second within cancer-related deaths in the United States $[1,2]$ and the fourth in China [3]. Therefore, finding new molecular biomarkers is necessary to improve the prognosis of CRC, as well as to create new treatment strategies and improve clinical outcome.

The Wnt and Notch signaling pathways have been shown to play a major role in intestinal morphogenesis and homeostasis [4-7]. The Wnt signaling pathway primarily regulates the self-renewal of the intestinal epithelium, and a high incidence of gastrointestinal malignancies might be induced by deregulation of this self-renewal processes [8]. Moreover, several members of the Wnt signaling pathway, either tumor suppressors APC and Axin 2 or oncogene $\beta$-catenin and lymphoid enhancer factor-1 (LEF1), are often aberrantly activated in CRC development [9]. The inappropriate activation of the Wnt signaling pathway and the subsequent formation of nuclear LEF/TCF/ $\beta$-catenin complexes lead to uncontrolled downstream target gene activation and ultimately result in the malignant transformation of cells [10]. In addition, transcription factor LEF1 is a potential candidate biomarker for CRC since it serves as a key role in the regulation of many important cellular functions, including proliferation [11], growth [12], survival [13], mobility [14], and angiogenesis [15].

The Notch signaling pathway maintains the balance between cell proliferation, differentiation, and apoptosis [16]. Furthermore, it has been reported that the Notch signaling pathway promotes cell survival, angiogenesis, and resistance 
TABLE 1: Results of LEF1 and Notch2 immunohistochemical analysis in tumor tissue in relation to the clinicopathologic characteristics of CRC patients and their tumors.

\begin{tabular}{|c|c|c|c|c|c|c|c|}
\hline \multirow{2}{*}{ Characteristic } & \multirow{2}{*}{$n$} & \multicolumn{2}{|c|}{ LEF1 expression } & \multirow{2}{*}{$P$} & \multicolumn{2}{|c|}{ Notch2 expression } & \multirow{2}{*}{$P$} \\
\hline & & Low & High & & Low & High & \\
\hline Total & 184 & 58 & 126 & & 120 & 64 & \\
\hline Age (years) & & & & 0.330 & & & 0.634 \\
\hline$<60$ & 72 & 26 & 46 & & 45 & 27 & \\
\hline$\geq 60$ & 112 & 32 & 80 & & 75 & 37 & \\
\hline Gender & & & & 0.339 & & & 0.640 \\
\hline Male & 104 & 36 & 68 & & 66 & 38 & \\
\hline Female & 80 & 22 & 58 & & 54 & 26 & \\
\hline Tumor location & & & & 0.858 & & & 0.200 \\
\hline Right colon & 40 & 12 & 28 & & 30 & 10 & \\
\hline Left colon & 46 & 16 & 30 & & 26 & 20 & \\
\hline Rectum & 98 & 30 & 68 & & 64 & 34 & \\
\hline Histology (differentiation) & & & & 0.071 & & & $<0.001$ \\
\hline Well & 86 & 24 & 62 & & 42 & 44 & \\
\hline Moderate & 68 & 28 & 40 & & 56 & 12 & \\
\hline Poor & 30 & 6 & 24 & & 22 & 8 & \\
\hline Node metastasis & & & & 0.001 & & & $<0.001$ \\
\hline$N_{0}$ & 80 & 36 & 44 & & 68 & 12 & \\
\hline$N_{1-3}$ & 104 & 22 & 82 & & 52 & 52 & \\
\hline Distant metastasis & & & & $<0.001$ & & & $<0.001$ \\
\hline No & 144 & 56 & 88 & & 84 & 60 & \\
\hline Yes & 40 & 2 & 38 & & 36 & 4 & \\
\hline TNM stage & & & & $<0.001$ & & & $<0.001$ \\
\hline I & 14 & 6 & 8 & & 4 & 10 & \\
\hline II & 56 & 12 & 44 & & 40 & 16 & \\
\hline III & 74 & 36 & 38 & & 42 & 32 & \\
\hline IV & 40 & 4 & 36 & & 34 & 6 & \\
\hline
\end{tabular}

to therapy in many different tumors [17]. The aberrant activation of Notch signaling has been associated with tumorigenesis; however, the exact function of Notch signaling in tumor development and progression remains unknown. Studies suggest that Notch signaling could be either oncogenic or antiproliferative depending upon the context of its activation [18]. In addition, both the Wnt and Notch signaling pathways have been reported to be useful therapeutic targets in several noncolorectal tumors $[19,20]$.

Based on these findings, the Wnt and Notch signaling pathways have been established as important key mediators of intestinal tumorigenesis. Nevertheless, the relationship between the combination of LEF1 status and Notch2 status and their prognostic relevance in CRC has yet to be established. Therefore, the aim of this study was to examine the potential crosstalk between these two pathways and their possible synergistic effects in tumorigenesis. We have evaluated the significance of LEF1 expression and Notch2 expression and their crosstalk in CRC. In addition, we have examined the association of these two potential biomarkers with the clinicopathologic characteristics and survival of patients.

\section{Materials and Methods}

2.1. Ethics Statement. Tissue collection was compliant with the agreement of the Conduct of Human Ethics Committee of the First Affiliated Hospital, College of Medicine of Xi'an Jiaotong University. Written informed consent was obtained from each patient.

2.2. Patients and Specimens. One-hundred and eighty-four patients with colorectal cancer who underwent curative surgery without chemotherapy and/or radiotherapy treatment at the First Affiliated Hospital, College of Medicine of Xi'an Jiaotong University from 2007 to 2008 were enrolled in this study. The study included 104 men and 80 women aged between 30 and 78 years (mean, 54 years). In addition to tumor tissue, 184 samples of matched paratumorous normal colorectal tissue (at $5 \mathrm{~cm}$ distance from the tumor) were taken from the same patients.

The main clinicopathologic data are presented in Table 1. The pathological types of all the specimens were confirmed by two independent pathologists under double-blinded conditions. Tumors were classified according to the criteria from the TNM Union for International Cancer Control (UICC), while tumor cellular differentiation (TCD) was defined by Edmondson's classification. The followup for all cases was terminated in February of 2013. During survival analysis, cases were regarded as censored data when patients were either lost to followup or died of causes other than CRC.

2.3. Immunohistochemistry. Formalin fixed paraffin-embedded sections were deparaffinized, rehydrated, and incubated 
with $3 \%$ hydrogen peroxidase. Next, the sections were heated in a microwave oven for $3 \mathrm{~min}$ at $100^{\circ} \mathrm{C}$ for antigen retrieval. Slides were then incubated with blocking serum and primary antibodies for LEF1 (1:100, C12A5; Cell Signaling Technology, Danvers, MA) and Notch2 (1:200, D76A6; Cell Signaling Technology, Danvers, MA), overnight at $4^{\circ} \mathrm{C}$. The immunohistochemical reaction was visualized with $0.05 \% \quad 3^{\prime}, 3^{\prime}$ diaminobenzidine tetrahydrochloride (DAB) followed by a counterstaining with hematoxylin. Finally, the sections were examined and analyzed using a microscope (Q550CW; Leica, Manheim, Germany). For the negative controls, the sections were incubated with preimmune rabbit serum instead of the primary antibodies. Immunostaining was defined independently by two pathologists blinded to the clinical data and scored by multiplying the intensity of staining and the percentage of the stained tumor cells. The intensity of staining was graded as follows: 0 (colorless), 1 (pallide-flavens), 2 (yellow), and 3 (brown). The percentage of the stained tumor cells was graded as $0(<5 \%), 1(5 \%-25 \%), 2(26 \%-50 \%), 3(51 \%-$ $75 \%)$ and 4 (>75\%). The final score was ranged from 0 to 12 . A score of 0 was defined as negative expression, scores 1-4 as "weak expression," scores 5-8 as "moderate expression," and scores 9-12 as "strong expression" [21]. For the purpose of further analysis, the samples with score $0-4$ were defined as low expression, while the samples with scores 5-12 were grouped and defined as high expression [22]. Few specimens with inconsistent score were re-evaluated again by two pathologists until the agreement was reached.

\subsection{Real-Time Reverse Transcription-Polymerase Chain Reac-} tion Analysis. The total mRNA was extracted from fresh tissue samples with TRIzol reagent according to the manufacturer's instruction (Invitrogen, Carlsbad, CA). Less than $500 \mathrm{ng}$ of total RNA were used for complementary DNA synthesis with an SYBR ExScript RT-PCR kit (Takara, Dalian, China). The reaction was performed using the iQ5 Multicolour Real-Time PCR Detection System (Bio-Rad, Hercules, CA) and SYBR Premix Ex Taq TM II (Takara). The primer sequences used for LEF1 and Notch2 analysis were as follows:

LEF1 forward $5^{\prime}$-AGCGAATGTCGTTGCTGAGTGTA-3', reverse $5^{\prime}$-CTCTTGCAGACCAGCCTGGATAA$3^{\prime}$, Notch2 forward $5^{\prime}$-CTACAGTTGTCGCTGCTTGC-3', reverse $5^{\prime}$-GTTGGAGAGGCACTCGTTGA-3' ${ }^{\prime}$, respectively. GAPDH was used as the internal housekeeping gene control, and the primer sequences were as follows: glyceraldehyde 3phosphate dehydrogenase (GAPDH) forward $5^{\prime}$-ATGGGGAAGGTGAAGGTCG-3', reverse $5^{\prime}$-GGGTCATTGATGGCAACAATATC-3'. For each real-time RT-PCR reaction, a dissociation curve analysis was performed with each reaction in triplicate. The data were acquired as a threshold cycle $\left(\Delta C_{t}\right)$ value. The $\Delta C_{t}$ values were determined by subtracting the average internal housekeeping gene $C_{t}$ value from the average target gene $C_{t}$ value. Since the amplification efficiency of the target genes and internal control gene was equal, the relative gene expression in the CRC tissues compared with paratumorous normal colorectal tissues was calculated using the $2^{-\Delta \Delta C_{t}}$ method, where $\Delta \Delta C_{t}=\Delta C_{t}$ (cancer tissue) $-\Delta C_{t}$ (paratumorous normal tissue).
2.5. Western Blot. The tissue samples used in Western blot analysis were lysed with cell lysis buffer as previously described [23]. Equal amounts of protein were separated by $6 \% \sim 12 \%$ SDS-PAGE and were electroblotted onto polyvinylidene difluoride membranes (Millipore, Danvers, MA), which were blocked with $5 \%$ blocking buffer and subsequently incubated with the following primary antibodies: anti-LEF1 antibody (1:800, C12A5; Cell Signaling Technology, Danvers, MA, USA), anti-Notch2 (1:800, D76A6; Cell Signaling Technology, Danvers, MA, USA), and anti- $\beta$-actin antibody (1:1000, sc-130301; Santa Cruz Biotechnology, Santa Cruz, CA, USA). Then, the membranes were incubated with a horseradish peroxidase-conjugated secondary antibody (Santa Cruz Biotechnology, Santa Cruz, CA,USA), and the blots were visualized using an ECL detection system (Millipore, Danvers, MA, USA). Qualitative analysis was used to define the negative expression (protein band, absent) or positive expression (protein band, present). Each experiment was performed three times.

2.6. Statistical Analysis. The associations between the LEF1 expression and Notch 2 expression and each clinicopathologic parameter were examined using either the $\chi^{2}$ test or Fisher's exact test (two sided). Student's $t$-tests (independent samples $t$-test) or a $u$-test were adopted to determine the difference between two sample means. One-way analysis of variance (ANOVA) was used to assess the difference among three or four sample means, and Spearman's rank test was used to assess the correlation between LEF1 expression and Notch2 expression. The survival rates were assessed by the KaplanMeier method and compared using the log-rank test. Multivariate analysis, using the Cox proportional hazards regression model, was performed to assess the prognostic value of the marker expression and clinicopathologic factors. All statistical analyses were performed using the SPSS 17.0 software (SPSS Inc., Chicago, USA). $P<0.05$ was considered to indicate statistical significance.

\section{Results}

3.1. Expression of LEF1 in CRC Tissues. We have examined the LEF1 expression in 184 primary CRC tissues and paired paratumorous normal colorectal tissues. Among these 184 primary CRC tissues, $126(68.5 \%)$ cases showed high LEF1 expression, while only $40(21.7 \%)$ cases in matched paratumorous normal colorectal tissues. Immunohistochemical staining revealed a predominantly nuclear localization of LEF1 (Figure 1(a)). The results of immunohistochemical analysis showed that LEF1 expression was significantly higher in CRC tissue than LEF1 expression in the paratumorous normal colorectal tissue $(P<0.001)$. In addition, significant differences in LEF1 expression in tumor tissue were observed between tumors with node metastasis, distant metastasis and different TNM stages $(P=0.001,<0.001,<0.001$, resp.) (Table 1). There was no significant association observed between the LEF1 expression and age, gender of patients, tumor location, or histology (Table 1). In addition to immunohistochemical analysis, real-time PCR analysis was used to 

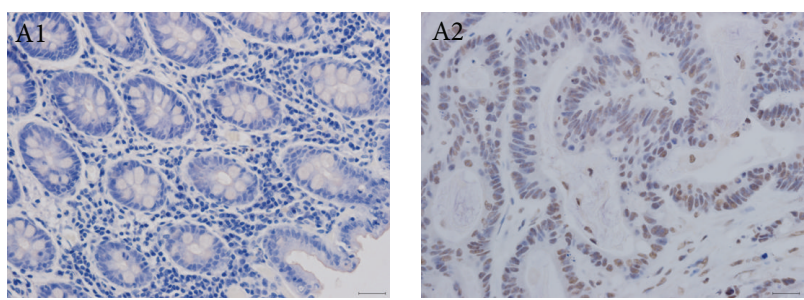

(a)
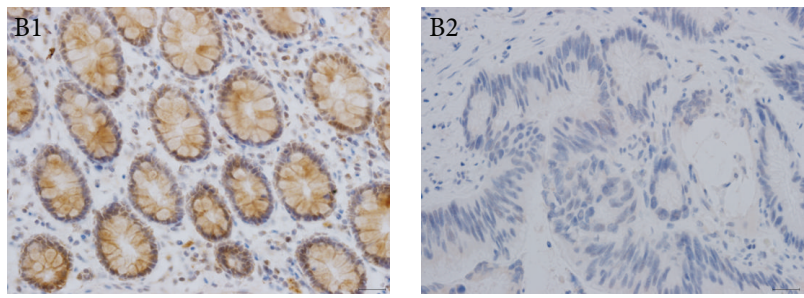
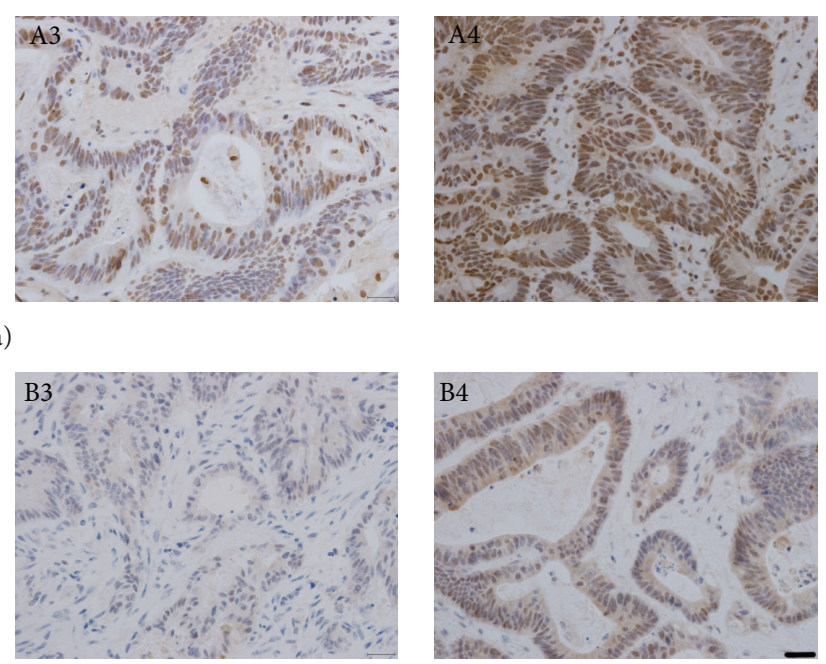

(b)

FIGURE 1: Immunohistochemical expression of LEF1 and Notch2 in CRC and paratumorous normal colorectal tissues. (a) Immunohistochemical staining of LEF1 expression in paratumorous normal colorectal tissues (A1) and CRC tissues (A2-A4), (A1) negative expression (score 0), (A2) weak expression (score 1-4), (A3) moderate expression (score 5-8), and (A4) strong expression (score 9-12). (b) Immunohistochemical staining of Notch2 expression in paratumorous normal colorectal tissues (B1) and CRC tissues (B2-B4), (B1) strong expression (score 9-12), (B2) negative expression (score 0), (B3) weak expression (score 1-4), and (B4) moderate expression (score 5-8); scale bars, $25 \mu \mathrm{m}$.

assess the LEF1 mRNA expression in 184 pairs of CRC tissues and paratumorous normal colorectal tissues. Our results showed that $L E F 1$ mRNA was significantly increased in most CRC tissues compared with the paratumorous normal colorectal tissues $(P<0.001)$, and the association between LEF1 mRNA expression and clinicopathologic factors was in accordance with the results of the immunohistochemical analysis (Table 2).

In addition, Western blot was used to confirm these results in the examined 184 paired tumor and corresponding normal tissues. The rate of positive LEF1 expression was $64.1 \%$ (118 out of 184) in CRC tissues and 20.1\% (37 out of 184) in the matched paratumorous normal colorectal tissues. LEF1 positive expression was significantly higher in CRC tissues than that in the matched paratumorous normal colorectal tissues $(P<0.01)$ (Figures 2(a) and 2(c)).

3.2. Expression of Notch 2 in CRC Tissues. As shown in Table 1 , only 64 out of $184(34.8 \%)$ analyzed cancer tissues showed high Notch2 protein expression. In contrast, high Notch2 expression was detected in 142 (77.2\%) out of 184 of paratumorous normal colorectal tissues, and Notch2 protein expression was observed in the membrane and/or cytoplasm of tissue cells (Figure $1(\mathrm{~b})$ ).

In our study, Notch2 expression presented a negative association with colorectal carcinomas $(P<0.001)$. Low Notch2 expression was strongly correlated with poor differentiation status, node metastasis, distant metastasis, and TNM stage $(P<0.001$, resp. $)$ (Table 1$)$.

In addition, Notch 2 mRNA levels were significantly decreased in most CRC tissues compared with paratumorous normal colorectal tissues $(P<0.001)$ (Table 2$)$. In Western blot analysis, positive Notch 2 expression was detected in only
40 out of $184(21.7 \%)$ analyzed cancer tissues compared with $73.9 \%$ (136 out of 184) in paratumorous normal colorectal tissues. Notch2 positive expression was significantly lower in cancer tissues than that in matched paratumorous normal colorectal tissue $(P<0.01)$ (Figures $2(\mathrm{~b})$ and $2(\mathrm{c}))$. Furthermore, Notch 2 mRNA expression was decreased in samples from patients with less differentiated tumors, node metastasis, distant metastasis, and of an advanced TNM stage $(P<$ 0.05 ) (Table 2), consistent with the results from immunohistochemical analysis.

3.3. Correlation between LEF1 Expression and Notch2 Expression in CRC. We analyzed the correlation between LEF1 expression and Notch2 expression in CRC at the protein level and mRNA level. Among the 184 analyzed CRC samples, 58 (31.5\%) were LEF1-low whereas 126 (68.5\%) were LEF1high tumors. In Notch2 immunohistochemical analysis, 120 (65.2\%) tumors were found to be Notch2 low, whereas 64 (34.8\%) were found to be Notch2 high (Table 1). A significant negative correlation between the LEF1 expression and Notch2 protein expression in the CRC samples was observed ( $r=$ $-0.315, P<0.001$, Spearman's rank test) (Figure 3). When analyzing Western blot data, we found a negative correlation between the LEF1 expression and Notch2 expression in the CRC tissues $(r=-0.430, P<0.001$, Spearman's rank test). A similar correlation between the LEF1 and Notch2 mRNA expression in the CRC samples was also observed $(r=$ $-0.571, P<0.001$, Spearman's rank test).

3.4. Survival Analysis. All 184 patients were included in the survival analysis (with followup period of 5 years) to assess LEF1 expression and Notch2 expression as potential prognostic factors in CRC. The survival time of patients included in 
TABLE 2: Results of LEF1 and Notch2 mRNA real-time PCR analysis in tumor tissue in relation to the clinicopathologic characteristics of CRC patients and their tumors.

\begin{tabular}{|c|c|c|c|c|c|}
\hline Characteristic & $n$ & LEF1, mean (SD) & $P$ & Notch2, mean (SD) & $P$ \\
\hline Tissue type & & & $<0.001$ & & $<0.001$ \\
\hline CRC tissues & 184 & $3.7270(1.7635)$ & & $0.5263(0.3320)$ & \\
\hline Paratumorous normal tissues & 184 & $0.8239(0.2624)$ & & $1.8675(0.4230)$ & \\
\hline Age (years) & & & 0.245 & & 0.292 \\
\hline$<60$ & 72 & $3.6175(1.5221)$ & & $0.5613(0.4012)$ & \\
\hline$\geq 60$ & 112 & $3.8617(1.1551)$ & & $0.5038(0.2875)$ & \\
\hline Gender & & & 0.879 & & 0.712 \\
\hline Male & 104 & $3.7410(1.6041)$ & & $0.5241(0.4057)$ & \\
\hline Female & 80 & $3.7088(1.2707)$ & & $0.5417(0.2362)$ & \\
\hline Tumor location & & & 0.611 & & 0.365 \\
\hline Right colon & 40 & $3.6889(1.3662)$ & & $0.5579(0.2331)$ & \\
\hline Left colon & 46 & $3.5371(1.4057)$ & & $0.5784(0.4124)$ & \\
\hline Rectum & 98 & $3.8317(1.8993)$ & & $0.4992(0.3346)$ & \\
\hline Histology (differentiation) & & & 0.609 & & $<0.001$ \\
\hline Well & 86 & $3.6022(1.9496)$ & & $0.6760(0.4661)$ & \\
\hline Moderate & 68 & $3.7840(1.6342)$ & & $0.4514(0.2112)$ & \\
\hline Poor & 30 & 3.9557 (1.5231) & & $0.3002(0.2214)$ & \\
\hline Node metastasis & & & $<0.001$ & & $<0.001$ \\
\hline$N_{0}$ & 80 & $2.2058(1.7023)$ & & $0.6510(0.4138)$ & \\
\hline$N_{1-3}$ & 104 & $4.8971(1.8106)$ & & $0.4304(0.2691)$ & \\
\hline Distant metastasis & & & $<0.001$ & & $<0.001$ \\
\hline No & 144 & $3.1840(1.7412)$ & & $0.6084(0.1059)$ & \\
\hline Yes & 40 & $5.6818(1.8334)$ & & $0.2557(0.5460)$ & \\
\hline TNM stage & & & $<0.001$ & & $<0.001$ \\
\hline I & 14 & $2.1012(1.7731)$ & & $0.8526(0.2113)$ & \\
\hline II & 56 & 2.9875 (1.2111) & & $0.6752(0.2547)$ & \\
\hline III & 74 & $3.3415(1.8521)$ & & $0.4369(0.1821)$ & \\
\hline IV & 40 & $6.0445(1.9694)$ & & $0.3940(0.6921)$ & \\
\hline
\end{tabular}

this study ranged from less than four months to more than 60 months, and the median survival time was 51 months. The analysis of prognostic factors for survival is summarized in Table 3.

3.5. Kaplan-Meier Univariate Survival Analysis of Clinicopathologic Factors and LEF1 Protein Expression and Notch2 Protein Expression. In order to assess the prognostic significance of the LEF1 and Notch2 protein expression, KaplanMeier survival curves were established (Figures 4(a) and $4(\mathrm{~b})$ ), and the results of the log-rank tests for the clinicopathologic factors and LEF1 protein expression and Notch2 expression in CRC patients are summarized in Table 3. In the univariate analysis, a statistically significant association with shorter survival time was observed for patients with poor tumor differentiation status, advanced TNM stage of tumors, and the combination of increased LEF1 coexpression and decreased Notch 2 coexpression $(P<0.05)$. The high LEF1 protein expression $[\operatorname{Exp}(B), 2.31 ; 95 \% \mathrm{CI}, 1.15-4.64 ; P=$ $0.016]$ and low Notch2 protein expression $[\operatorname{Exp}(B), 2.26 ; 95 \%$ CI, 1.15-4.43; $P=0.017$ ] were also proved to be associated with shorter survival and higher risk of death in patients with CRC. However, gender $(P=0.909)$, age $(P=0.698)$ or tumor location $(P=0.644 ; P=0.587)$ had no prognostic value on survival of patients with CRC.

3.6. Multivariate Survival Analysis of Clinicopathologic Factors and LEF1 Protein Expression and Notch2 Protein Expression. In multivariate analysis, the Cox proportional hazards model was adjusted for gender, age, differentiation status, TNM stage, and LEF1 expression and Notch2 expression. As a result, LEF1 and Notch2 protein levels proved to be independent predictors of survival for patients with CRC, indicating that patients with a high LEF1 expression and low Notch2 expression had a higher risk of death than those with LEF1 (low)/Notch2 (high) tumors. Moreover, TNM stage and the combination of increased LEF1 coexpression and decreased Notch2 coexpression also proved to be prognostic factor for CRC patients $(P<0.05)$ (Table 3$)$. There was no significant association found with the overall survival for other analyzed clinicopathologic factors.

3.7. Prognostic Significance of the Combined LEF1 Protein Expression and Notch 2 Protein Expression Profile. The present results showed that LEF1 expression was higher in CRC 

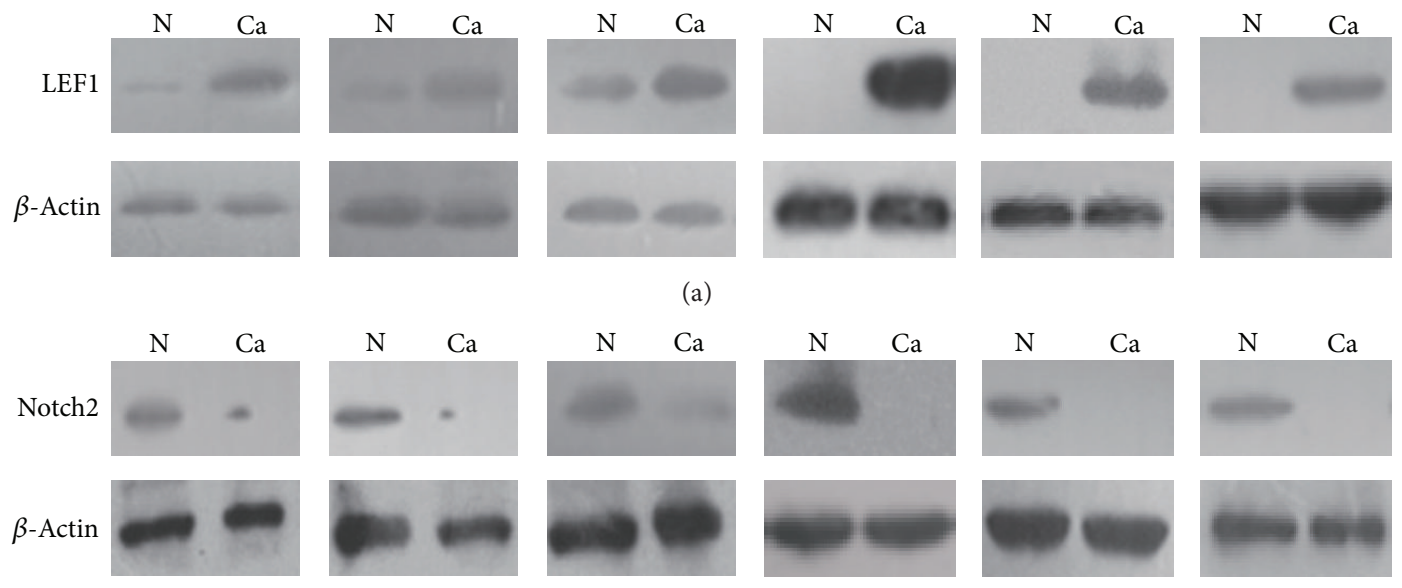

(a)

(b)
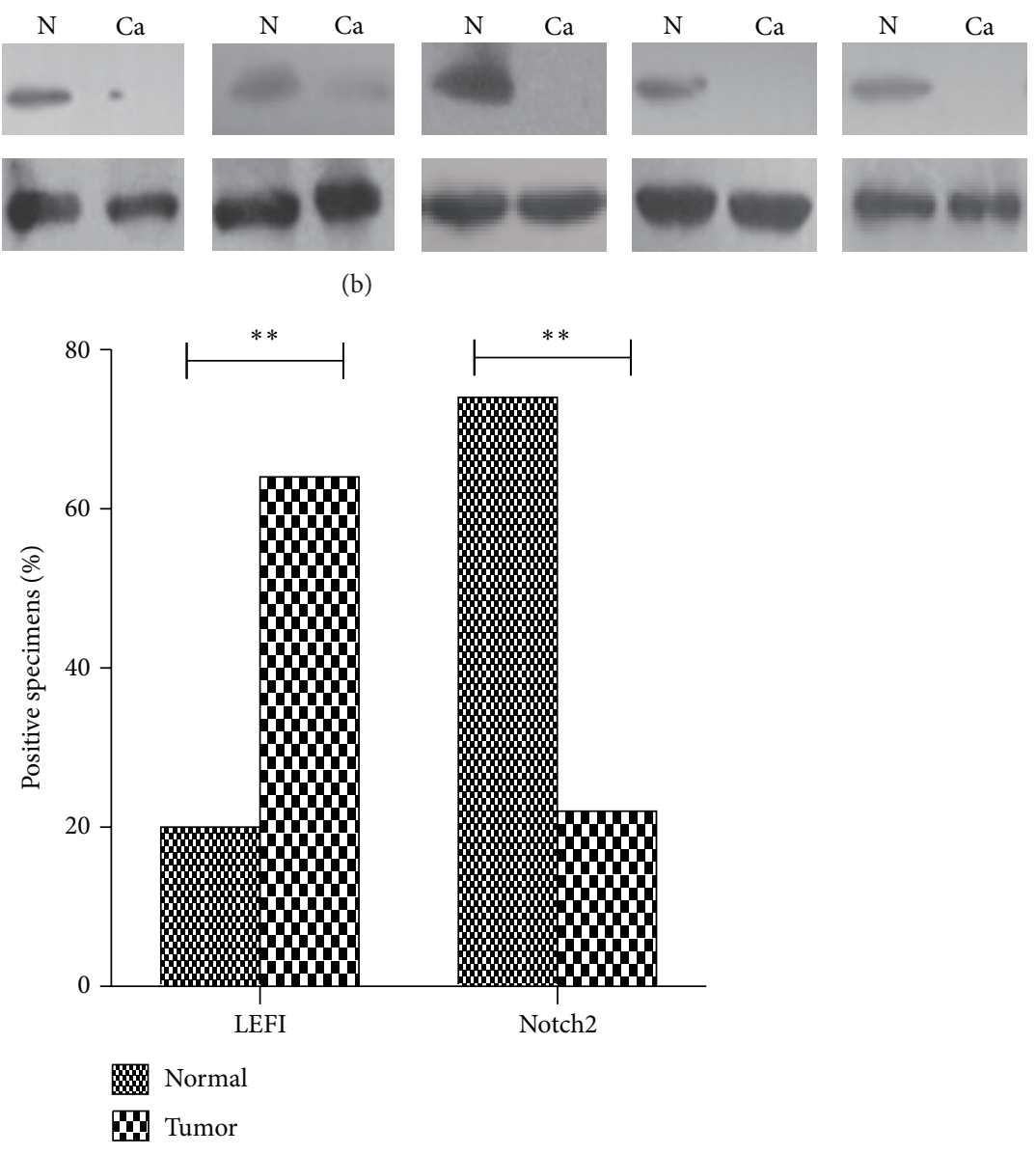

(c)

FIGURE 2: Western blot analysis of LEF1 and Notch2 in CRC tissues and paratumorous normal colorectal tissues. $\beta$-actin was used as an internal control. (a) LEF1 expression in CRC tissues and the corresponding paratumorous normal colorectal tissues of the same patient. (b) Notch2 expression in CRC tissues and corresponding paratumorous normal colorectal tissues of the same patient. (c) The percentage of LEF1 and Notch2 positive specimens in CRC tissues (tumor) and corresponding paratumorous normal colorectal tissues (normal). $\chi^{2}$ test was used for statistical analyses. ${ }^{* *} P<0.01$.

tissues compared to the corresponding paratumorous normal tissue, whereas Notch2 expression was lower in the tumor tissue than in the matching normal colorectal tissue. These results indicated that LEF1 and Notch2 might present an opposite function during the development of CRC. In addition, the Spearman's rank test showed that there was a significant negative correlation between LEF1 expression and Notch2 expression in these tumors.

In order to estimate the prognostic significance of the combined LEF1 and Notch2 expression profile and to detect the LEF1 expression and Notch2 expression associations with overall survival, we have reclassified patients into the following four groups: LEF1 (low)/Notch2 (high) $(n=33)$, LEF1 (high)/Notch2 (high) $(n=31)$, LEF1 (low)/Notch2 (low) $(n=$ 25), and LEF1 (high)/Notch2 (low) $(n=95)$. The subgroup analysis showed that patients with an LEF1 high expression and Notch2 low expression had a shorter overall survival time than all other combined status patients (Figures 4(c) and 4(d)). Multivariate analysis revealed that patients with high LEF1 expression and low Notch2 expression tumors had a significantly worse overall survival compared with patients of other combined LEF1/Notch2 expression groups (Table 3). 


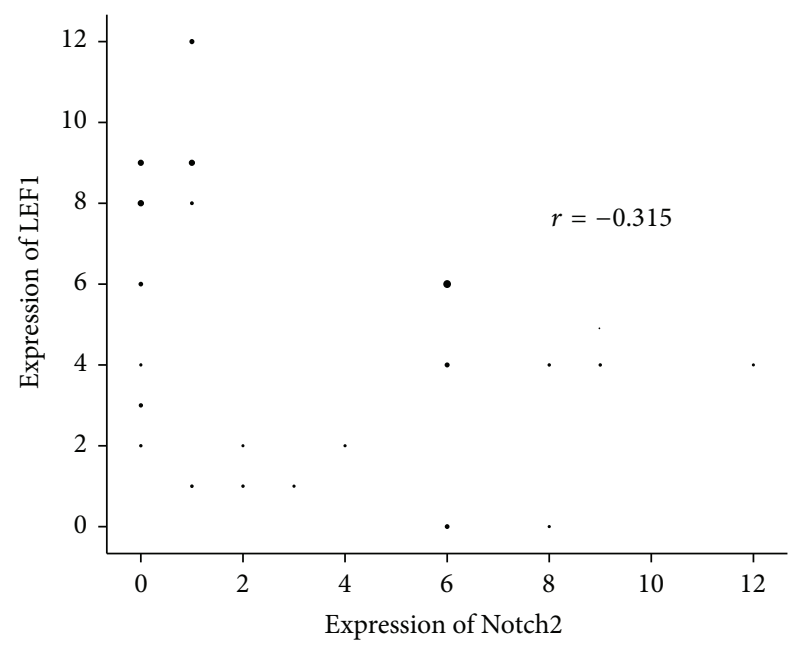

FIgURE 3: A correlation between LEF1 immunohistochemical expression and Notch2 immunohistochemical expression in CRC. The dot figure showed a negative correlation between LEF1 expression and Notch2 expression (Spearman's rank test $r=-0.315, P<$ 0.001). $X$-axis: Notch2 expression level of each patient; $Y$-axis: LEF1 expression level of each patient. Some of the data was overlapping. The size of the points reflected the number of data points in each location (i.e., larger points reflect a greater number of data points at a given location).

\section{Discussion}

In this study, we aimed to examine the expression of LEF1 and Notch2 in CRC and their association with clinicopathologic variables and CRC patients' overall survival; therefore, an appropriate IHC scoring system and statistical method were very important for our study. We semiquantified the IHC staining for LEF1 and Notch2 ranging from 0 to 12, then classified score $0-4$ as low expression and 5-12 as high expression and used these re-converted enumeration data for statistical analyses. We thought that the enumeration data were more suitable for the comparison between kinds of groups, especially for statistical analysis with respect to overall survival. There are several similar literature reports which use the evaluation of the converted IHC staining results before starting the statistical analysis, compared with the use of final score [24-26]. Our results indicate that $L E F 1 \mathrm{mRNA}$ and protein expression were increased in CRC tissue and were correlated with the node metastasis, distant metastasis and the TNM stage of tumors. Most importantly, a statistically significant relation was observed between the LEF1 protein expression and patients' survival, and those whose tumors were high LEF1 expression had a shorter survival time. Opposite to our findings, in the study by Kriegl et al., higher LEF1 expression was associated with longer patient survival time [27]. In the study of Chu et al. [28], low Notch2 expression was associated with unfavorable clinicopathologic features and a poor prognosis of CRC patients, consistent with our data. Nevertheless, they did not compare it with the LEF1 expression. The significantly different outcomes between patients expressing high and low levels of LEF1 and Notch2 suggested that LEF1 and Notch2 may be used to predict the clinical outcome.
These findings are valuable in the providing potential therapeutic targets for the future treatment of CRC.

It has been shown that LEF1 is critical for the adhesion and/or migration of tumor cells, indicating that LEF1 is probably involved in tumor invasion and metastasis [29]. LEF1 protein belongs to a high mobility group (HMG) family, which has been implicated in DNA binding $[30,31]$. In addition, it plays a pivotal role in carcinogenesis and the progression of CRC partly due to its involvement in the LEF1/ $\beta$ catenin complex, a crucial effector of the Wnt signaling pathway. Our study established that increased LEF1 expression was correlated with node metastasis, distant metastasis, and the advanced TNM stage of tumors. These results have proven that LEF1 is involved in the invasion and metastasis of CRC, consistent with some early findings of other authors [32-34].

The Notch signaling pathway plays an essential role in the differentiation of the gastrointestinal tract $[6,35,36]$ and can either have oncogenic or tumor suppressor functions in different cancers. Notch2 is one of the Notch receptors, which interacts with the DSL (Delta/Serrate/Lag-2) family of ligands to regulate the cell differentiation [37]. Notch2 has the highest homology with Notch1, but unlike Notch1, it can present a tumor-suppressive action in breast cancer $[38,39]$. It has recently been reported that Notch2 is a novel target for $\beta$-catenin-dependent Wnt signaling [40]. In our study, low Notch 2 mRNA and protein expression correlated with poor differentiation status, node metastasis, distant metastasis, and the advanced TNM stage in CRC. Therefore, a loss of Notch2 is a general feature during CRC progression, indicating a potentially conflicting role of LEF1 and Notch2 in this type of cancer.

It is known that alterations in the Wnt and Notch signaling pathways play a significant role in numerous cancers. Studies have shown that these two pathways are aberrantly activated in CRC [21, 28, 29]; however, the possible crosstalk between these two pathways in cancer development is unknown. Although an increasing body of evidence indicates that Wnt and Notch pathways crosstalk and transactivate each other in the normal tissue development as well as in cancer [41], such as in the survival of murine T-cell lymphomas [42], the mechanism by which Wnt pathway transactivates Notch pathway has not yet been fully determined. Therefore, further studies are necessary to disclose the possible convergent points of these two pathways in order to completely understand the relationship between the Wnt and Notch pathways in tumorigenesis.

In our study, we have examined the LEF1 and Notch2 mRNA and protein expression levels in a collection of 184 CRC patients stratified according to their outcomes. We showed the first, direct evidence of a negative correlation between LEF1 expression and Notch2 expression, as well as a high LEF1 expression and low Notch2 expression in relation with malignant CRC transformation. Moreover, high LEF1 protein expression and low Notch2 protein expression indicated a poorer prognosis in CRC. In viewing these results, it is understood that LEF1 has an oncogenic and Notch2 a tumor suppressor role during the development of CRC, consistent with the results of previous studies [42-44]. The possible reason for the functional diversity of LEF1 and Notch2 might 
TABLE 3: Association of LEF1/Notch2 and clinical factors with overall survival.

\begin{tabular}{|c|c|c|c|c|c|}
\hline Clinicopathologic characteristics & $n$ & Risk ratio $(95 \% \mathrm{CI})$ & $P$ & Risk ratio $^{\mathrm{b}}(95 \% \mathrm{CI})$ & $P$ \\
\hline \multicolumn{6}{|l|}{ LEF1 } \\
\hline Low & 58 & - & & - & \\
\hline High & 126 & $2.31(1.15-4.64)$ & 0.016 & $2.67(1.31-4.85)$ & $<0.001$ \\
\hline \multicolumn{6}{|l|}{ Notch2 } \\
\hline High & 64 & - & & - & \\
\hline Low & 120 & $2.26(1.15-4.43)$ & 0.017 & $2.48(1.22-4.80)$ & $<0.001$ \\
\hline \multicolumn{6}{|l|}{ Age } \\
\hline$<60$ & 72 & - & & - & \\
\hline$\geq 60$ & 112 & $0.44(0.23-0.87)$ & 0.698 & $0.35(0.12-1.01)$ & 0.053 \\
\hline \multicolumn{6}{|l|}{ Gender } \\
\hline Female & 104 & - & & - & \\
\hline Male & 80 & $0.98(0.55-1.71)$ & 0.909 & $1.01(0.97-1.06)$ & 0.532 \\
\hline \multicolumn{6}{|l|}{ Tumor location } \\
\hline Right colon & 40 & - & & - & \\
\hline Left colon & 46 & $1.17(0.59-2.37)$ & 0.644 & $0.65(0.13-1.27)$ & 0.604 \\
\hline Rectum & 98 & $0.79(0.34-1.83)$ & 0.587 & $0.63(0.18-1.25)$ & 0.476 \\
\hline \multicolumn{6}{|l|}{ Histology (differentiation) } \\
\hline Well & 86 & - & & - & \\
\hline Moderate & 68 & $1.45(1.20-1.98)$ & 0.001 & $0.57(0.19-1.69)$ & 0.313 \\
\hline Poor & 30 & $2.72(2.11-3.42)$ & $<0.001$ & $0.55(0.20-1.47)$ & 0.231 \\
\hline \multicolumn{6}{|l|}{ TNM stage } \\
\hline I & 14 & - & & - & \\
\hline II & 56 & $1.32(0.29-6.14)$ & 0.719 & $1.19(0.72-1.72)$ & 0.256 \\
\hline III & 74 & $2.88(1.06-7.28)$ & 0.038 & $2.85(1.13-7.44)$ & 0.030 \\
\hline IV & 40 & $2.76(1.38-5.51)$ & 0.004 & $2.83(1.68-4.74)$ & $<0.001$ \\
\hline \multicolumn{6}{|l|}{ LEF1 and Notch2 } \\
\hline Other combinations & 89 & - & & - & \\
\hline LEF1 (H)/Notch2 (L) & 95 & $3.40(1.90-7.25)$ & 0.006 & $2.66(1.98-3.32)$ & $<0.001$ \\
\hline
\end{tabular}

${ }^{\mathrm{a}}$ Unadjusted risk ratio in univariate models.

${ }^{\mathrm{b}}$ Adjusted risk ratio in multivariate models.

CI: confidence interval.

L: low expression; H: high expression.

be in the regulation of different upstream proteins. Ungerbäck et al. [40] have reported increased Notch2 promoter activity upon the cotransfection of colon cancer cells with high expression recombinant LEF1. Based on these findings, we assumed that LEF1 either directly regulates Notch2 or another protein acting as a bridge, which then activates LEF1 and inhibits Notch2 activity. Nevertheless, the exact molecular mechanism of these processes will need to be determined in future studies.

We further examined whether the expression of LEF1 and Notch2 was associated with the survival of CRC patients. Although both LEF1 and Notch2 protein expression were independent prognostic factors, they had completely opposite effects on survival. Patients with a high LEF1 protein expression had a worse outcome prognosis than those with a low LEF1 expression, consistent with the oncogenic role of LEF1.

We have also investigated the protein expression pattern of Notch2 and opposite to LEF1 findings, patients with a loss of Notch2 expression had a higher risk of death than those with a higher Notch2 expression. Based on our results, it seems that either low LEF1 expression or high Notch2 expression might be predictors of good survival outcomes in CRC patients. Furthermore, we showed that the abnormal coexpression of LEF1/Notch2 was a more efficient predictor than any LEF1 expression or Notch2 expression when separately analyzed. The abnormal coexpression of LEF1 (high)/Notch2 (low) was correlated with the advanced TNM stage of tumors. Also, a worse disease outcome and an extremely poor survival rate were observed in patients with LEF (high)/Notch2 (low) tumors when compared to patients whose tumors showed either LEF1 high expression or Notch2 low expression. These findings imply that a combination of LEF1 expression and Notch2 expression could be an effective molecular prognostic marker in CRC. To our knowledge, this is the first study to demonstrate these findings.

In conclusion, we have shown the possible coregulation of Wnt and Notch signaling pathways through the association of LEF1 expression and Notch2 expression with some of the CRC clinical characteristics and their opposite prognostic roles in CRC. This combined status could potentially be used as an even more efficient prognostic predictor in CRC patients. Based on our results, we conclude that LEF1 and Notch2 play key roles in CRC tumorigenesis and could potentially represent new targets for the design of new antitumor therapies. 


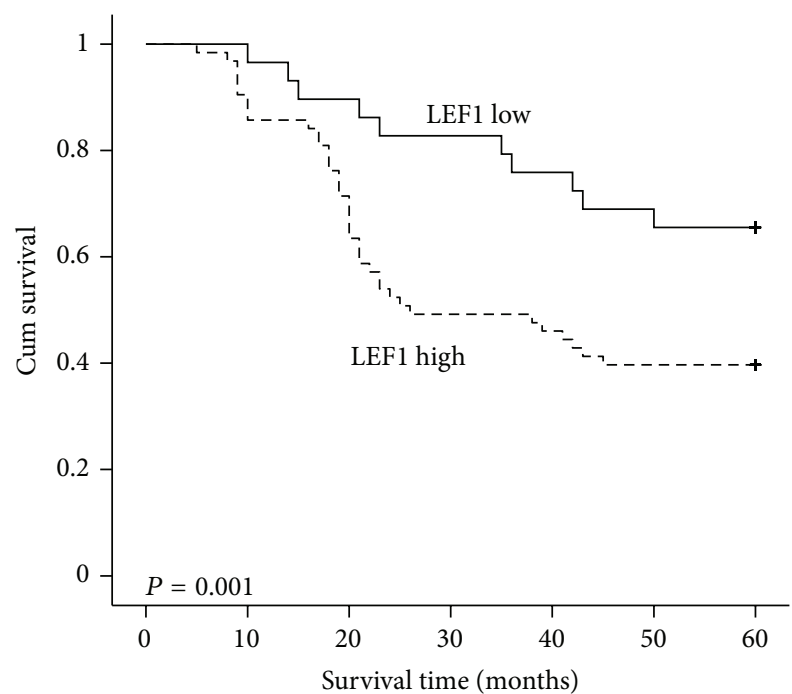

(a)

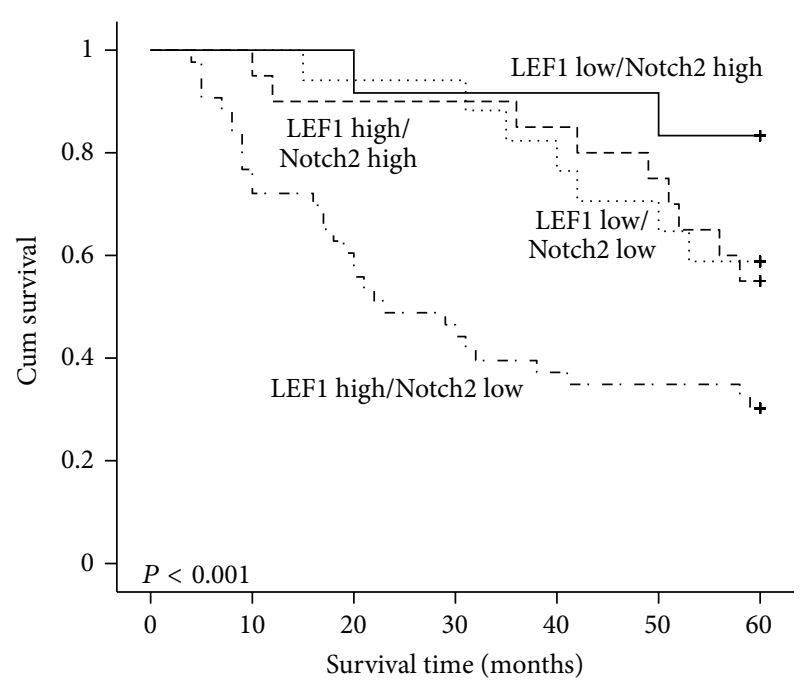

(c)

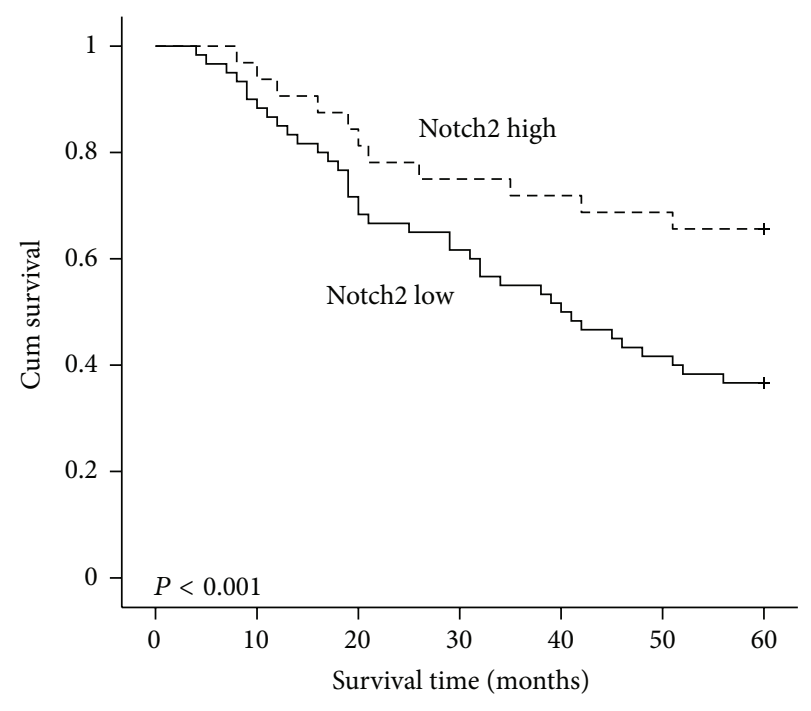

(b)

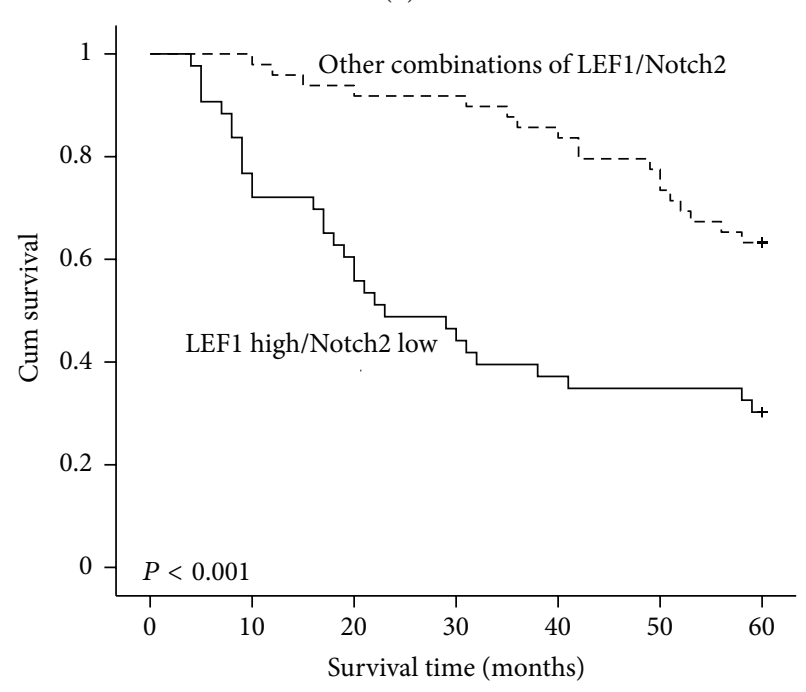

(d)

FIGURE 4: Kaplan-Meier survival curves showing significantly different survival rates for patients with CRC according to LEF1 expression and Notch2 expression. (a) Survival curves for LEF1; (b) Survival curves for Notch2; (c) survival curves for all patients divided by combination of LEF1 and Notch2 status; (d) survival curves for LEF1 high/Notch2 low and other combinations of LEF1/Notch2 expression.

\section{Conflict of Interests}

The authors declare that they have no conflict of interests.

\section{Authors' Contribution}

Wen-Juan Wang and Yu Yao are equal contributors.

\section{Acknowledgments}

This work was financially supported by grants from the National Natural Science Foundation of China (no. 81001090), Basical Research Foundation of Higher Education of China (no. xjj2010013), and Specialized Research Fund for the Doctoral Program of Higher Education of China (no.
20110201120061). The authors are grateful to Proffessor WeiXi Wang (Xian Jiaotong University, Xi'an, China) for providing useful comments on writing this paper.

\section{References}

[1] T. Gansler, P. A. Ganz, M. Grant et al., "Sixty years of ca: a cancer journal for clinicians," CA Cancer Journal for Clinicians, vol. 60, no. 6, pp. 345-350, 2010.

[2] A. Jemal, R. Siegel, J. Xu, and E. Ward, "Cancer statistics, 2010," CA Cancer Journal for Clinicians, vol. 60, no. 5, pp. 277-300, 2010.

[3] T. Lei, W.-Q. Chen, S.-W. Zhang et al., "Prevalence trend of colorectal cancer in 10 cities and counties in China from 1988 to 2002," Zhonghua Zhong Liu Za Zhi, vol. 31, no. 6, pp. 428-433, 2009. 
[4] P. Andreu, S. Colnot, C. Godard et al., "Crypt-restricted proliferation and commitment to the Paneth cell lineage following Apc loss in the mouse intestine," Development, vol.132, no. 6, pp. 1443-1451, 2005.

[5] S. Fre, M. Huyghe, P. Mourikis, S. Robine, D. Louvard, and S. Artavanis-Tsakonas, "Notch signals control the fate of immature progenitor cells in the intestine," Nature, vol. 435, no. 7044, pp. 964-968, 2005.

[6] S. Fre, S. K. Pallavi, M. Huyghe et al., "Notch and Wnt signals cooperatively control cell proliferation and tumorigenesis in the intestine," Proceedings of the National Academy of Sciences of the United States of America, vol. 106, no. 15, pp. 6309-6314, 2009.

[7] J. H. van Es, M. E. van Gijn, O. Riccio et al., "Notch $/ \gamma$-secretase inhibition turns proliferative cells in intestinal crypts and adenomas into goblet cells," Nature, vol. 435, no. 7044, pp. 959-963, 2005.

[8] J. H. Van Es and H. Clevers, "Notch and Wnt inhibitors as potential new drugs for intestinal neoplastic disease," Trends in Molecular Medicine, vol. 11, no. 11, pp. 496-502, 2005.

[9] M. Shitashige, S. Hirohashi, and T. Yamada, "Wnt signaling inside the nucleus," Cancer Science, vol. 99, no. 4, pp. 631-637, 2008.

[10] T.-W. Li, J.-H. Ting, N. N. Yokoyama, A. Bernstein, M. Van De Wetering, and M. L. Waterman, "Wnt activation and alternative promoter repression of LEF1 in colon cancer," Molecular and Cellular Biology, vol. 26, no. 14, pp. 5284-5299, 2006.

[11] D. N. Shelton, H. Fornalik, T. Neff et al., "The role of LEF1 in endometrial gland formation and carcinogenesis," Plos One, vol. 7, Article ID e40312, 2012.

[12] L. E. Valdivia, R. M. Young, T. A. Hawkins et al., "Lef1dependent $\mathrm{Wnt} / \beta$-catenin signalling drives the proliferative engine that maintains tissue homeostasis during lateral line development," Development, vol. 138, no. 18, pp. 3931-3941, 2011.

[13] K. H. Metzeler, B. Heilmeier, K. E. Edmaier et al., "High expression of lymphoid enhancer-binding factor-1 (LEF1) is a novel favorable prognostic factor in cytogenetically normal acute myeloid leukemia," Blood, vol. 120, pp. 2118-2126, 2012.

[14] L. H. Hoeppner, F. J. Secreto, D. F. Razidlo, T. J. Whitney, and J. J. Westendorf, "Lef1 $\Delta \mathrm{N}$ binds $\beta$-catenin and increases osteoblast activity and trabecular bone mass," Journal of Biological Chemistry, vol. 286, no. 13, pp. 10950-10959, 2011.

[15] L.-K. Phng, M. Potente, J. D. Leslie et al., "Nrarp coordinates endothelial Notch and Wnt signaling to control vessel density in angiogenesis," Developmental Cell, vol. 16, no. 1, pp. 70-82, 2009.

[16] T. K. Noah and N. F. Shroyer, "Notch in the intestine: regulation of homeostasis and pathogenesis," Annual Review of Physiology, vol. 75, pp. 263-288, 2013.

[17] J.-L. Li, R. C. Sainson, C. E. Oon et al., "DLL4-Notch signaling mediates tumor resistance to anti-VEGF therapy in vivo," Cancer Research, vol. 71, no. 18, pp. 6073-6083, 2011.

[18] G. P. Dotto, "Notch tumor suppressor function," Oncogene, vol. 27, no. 38, pp. 5115-5123, 2008.

[19] A. Pannuti, K. Foreman, P. Rizzo et al., "Targeting Notch to target cancer stem cells," Clinical Cancer Research, vol. 16, no. 12, pp. 3141-3152, 2010.

[20] T. Sasaki, H. Suzuki, K. Yagi et al., "Lymphoid enhancer factor 1 makes cells resistant to transforming growth factor $\beta$-induced repression of c-myc," Cancer Research, vol. 63, no. 4, pp. 801806, 2003.
[21] D. Chu, J. Zheng, W. Wang et al., "Notch2 expression is decreased in colorectal cancer and related to tumor differentiation status," Annals of Surgical Oncology, vol. 16, no. 12, pp. 32593266, 2009.

[22] X. Liu, S. Wang, X. Xia et al., "Synergistic role between p53 and JWA: prognostic and predictive biomarkers in gastric cancer," Plos One, vol. 7, Article ID e52348, 2012.

[23] T. Tian, K.-J. Nan, S.-H. Wang et al., "PTEN regulates angiogenesis and VEGF expression through phosphatase-dependent and -independent mechanisms in HepG2 cells," Carcinogenesis, vol. 31, no. 7, pp. 1211-1219, 2010.

[24] M. Barbareschi, P. Maisonneuve, D. Aldovini et al., "High syndecan-1 expression in breast carcinoma is related to an aggressive phenotype and to poorer prognosis," Cancer, vol. 98, no. 3, pp. 474-483, 2003.

[25] X. Liu, H. Zhang, L. Lai et al., "Ribonucleotide reductase small subunit M2 serves as a prognostic biomarker and predicts poor survival of colorectal cancers," Clinical Science, vol. 124, no. 9, pp. 567-578, 2013.

[26] W. Weichert, A. Röske, V. Gekeler et al., "Association of patterns of class I histone deacetylase expression with patient prognosis in gastric cancer: a retrospective analysis," The Lancet Oncology, vol. 9, no. 2, pp. 139-148, 2008.

[27] L. Kriegl, D. Horst, J. A. Reiche, J. Engel, T. Kirchner, and A. Jung, "LEF-1 and TCF4 expression correlate inversely with survival in colorectal cancer," Journal of Translational Medicine, vol. 8, article 123, 2010.

[28] D. Chu, Z. Zhang, Y. Zhou et al., "Notch1 and Notch2 have opposite prognostic effects on patients with colorectal cancer," Annals of Oncology, vol. 22, no. 11, pp. 2440-2447, 2011.

[29] A.-Y. Lin, M.-S. Chua, Y.-L. Choi et al., "Comparative profiling of primary colorectal carcinomas and liver metastases identifies LEF1 as a prognostic biomarker," PLoS One, vol. 6, no. 2, Article ID e16636, 2011.

[30] K. Giese, A. Amsterdam, and R. Grosschedl, "DNA-binding properties of the HMG domain of the lymphoid-specific transcriptional regulator LEF-1," Genes and Development, vol. 5, no. 12, pp. 2567-2578, 1991.

[31] A. Milatovich, A. Travis, R. Grosschedl, and U. Francke, "Gene for lymphoid enhancer-binding factor 1 (LEF1) mapped to human chromosome 4 (q23-q25) and mouse chromosome 3 near Egf," Genomics, vol. 11, no. 4, pp. 1040-1048, 1991.

[32] K. Hovanes, T.-W. Li, J. E. Munguia et al., “ $\beta$-Cateninsensitive isoforms of lymphoid enhancer factor-1 are selectively expressed in colon cancer," Nature Genetics, vol. 28, no. 1, pp. 5357, 2001.

[33] T. Reya and H. Clevers, "Wnt signalling in stem cells and cancer," Nature, vol. 434, no. 7035, pp. 843-850, 2005.

[34] R. T. Moon, A. D. Kohn, G. V. De Ferrari, and A. Kaykas, "WNT and $\beta$-catenin signalling: diseases and therapies," Nature Reviews Genetics, vol. 5, no. 9, pp. 691-701, 2004.

[35] Å. Apelqvist, H. Li, L. Sommer et al., "Notch signalling controls pancreatic cell differentiation," Nature, vol. 400, no. 6747, pp. 877-881, 1999.

[36] S. Miyamoto and D. W. Rosenberg, "Role of Notch signaling in colon homeostasis and carcinogenesis," Cancer Science, vol. 102, no. 11, pp. 1938-1942, 2011.

[37] S.-Y. Lee, K. Kumano, K. Nakazaki et al., "Gain-of-function mutations and copy number increases of Notch2 in diffuse large B-cell lymphoma," Cancer Science, vol. 100, no. 5, pp. 920-926, 2009. 
[38] C. F. O'Neill, S. Urs, C. Cinelli et al., "Notch2 signaling induces apoptosis and inhibits human MDA-MB-231 xenograft growth," American Journal of Pathology, vol. 171, no. 3, pp. 1023-1036, 2007.

[39] C. Parr, G. Watkins, and W.-G. Jiang, "The possible correlation of Notch-1 and Notch-2 with clinical outcome and tumour clinicopathological parameters in human breast cancer," International Journal of Molecular Medicine, vol. 14, no. 5, pp. 779-786, 2004.

[40] J. Ungerbäck, N. Elander, J. Grünberg, M. Sigvardsson, and P. Söderkvist, "The Notch-2 gene is regulated by Wnt signaling in cultured colorectal cancer cells," PLoS One, vol. 6, no. 3, Article ID e17957, 2011.

[41] F. E. Bertrand, C. W. Angus, W. J. Partis, and G. Sigounas, "Developmental pathways in colon cancer: crosstalk between WNT, BMP, Hedgehog and Notch," Cell Cycle, vol. 11, no. 23, pp. 4344-4351, 2012.

[42] C. Spaulding, E. J. Reschly, D. E. Zagort et al., "Notchl co-opts lymphoid enhancer factor 1 for survival of murine T-cell lymphomas," Blood, vol. 110, no. 7, pp. 2650-2658, 2007.

[43] X. Fan, I. Mikolaenko, I. Elhassan et al., "Notch1 and Notch2 have opposite effects on embryonal brain tumor growth," Cancer Research, vol. 64, no. 21, pp. 7787-7793, 2004.

[44] S.-H. Wang, K.-J. Nan, Y.-C. Wang, W.-J. Wang, and T. Tian, "The balance between two isoforms of LEF-1 regulates colon carcinoma growth," BMC Gastroenterol, vol. 12, article 53, 2012. 


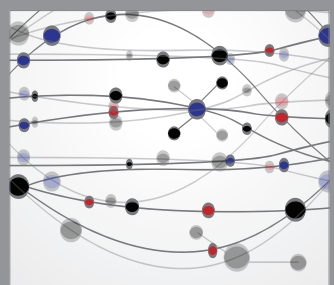

The Scientific World Journal
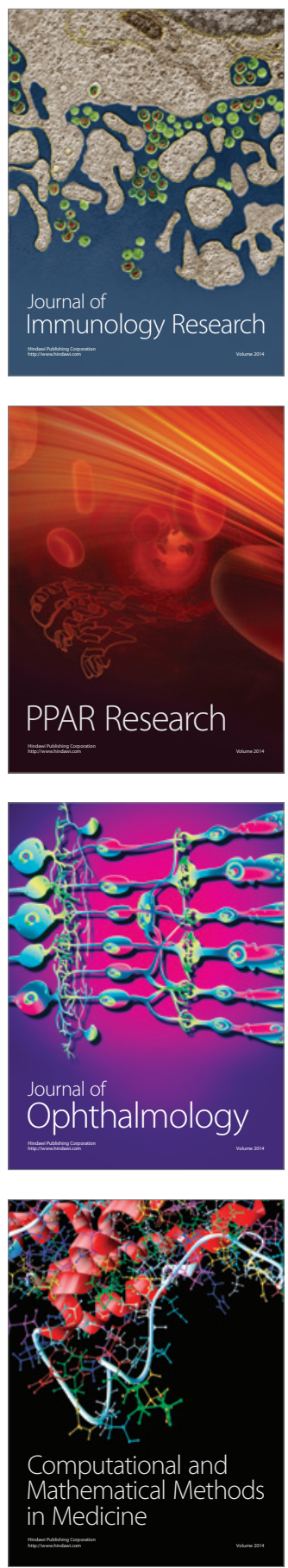

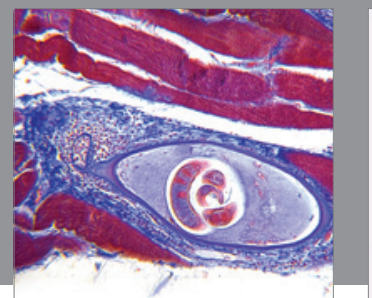

Gastroenterology

Research and Practice
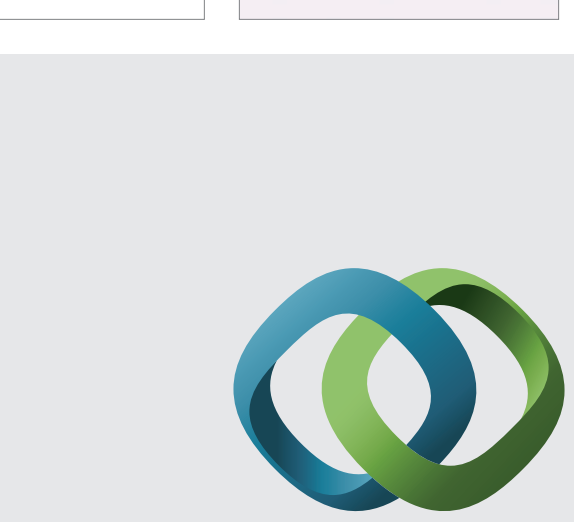

\section{Hindawi}

Submit your manuscripts at

http://www.hindawi.com
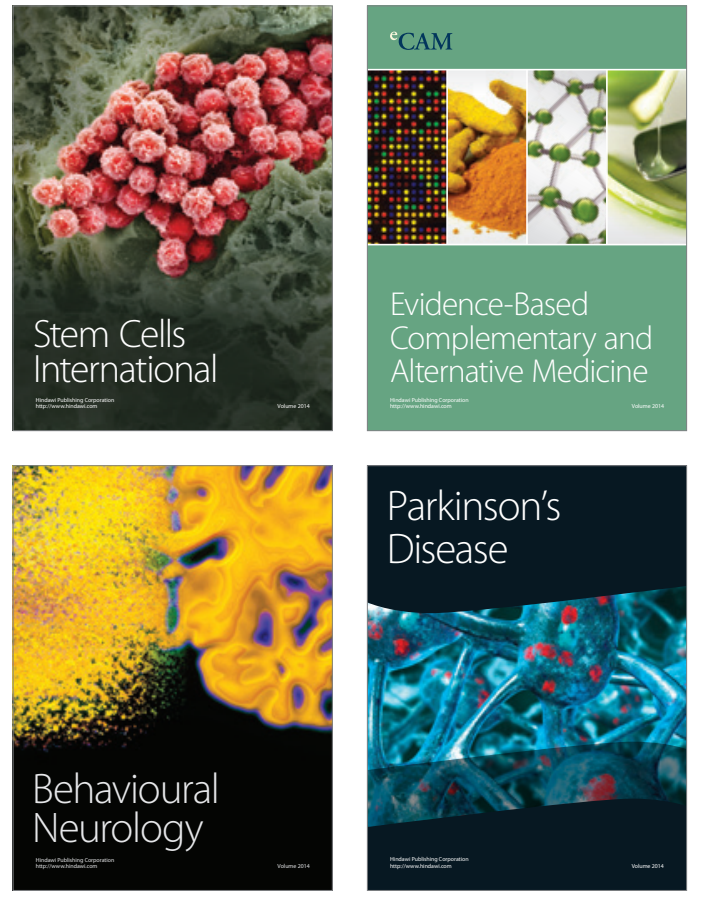
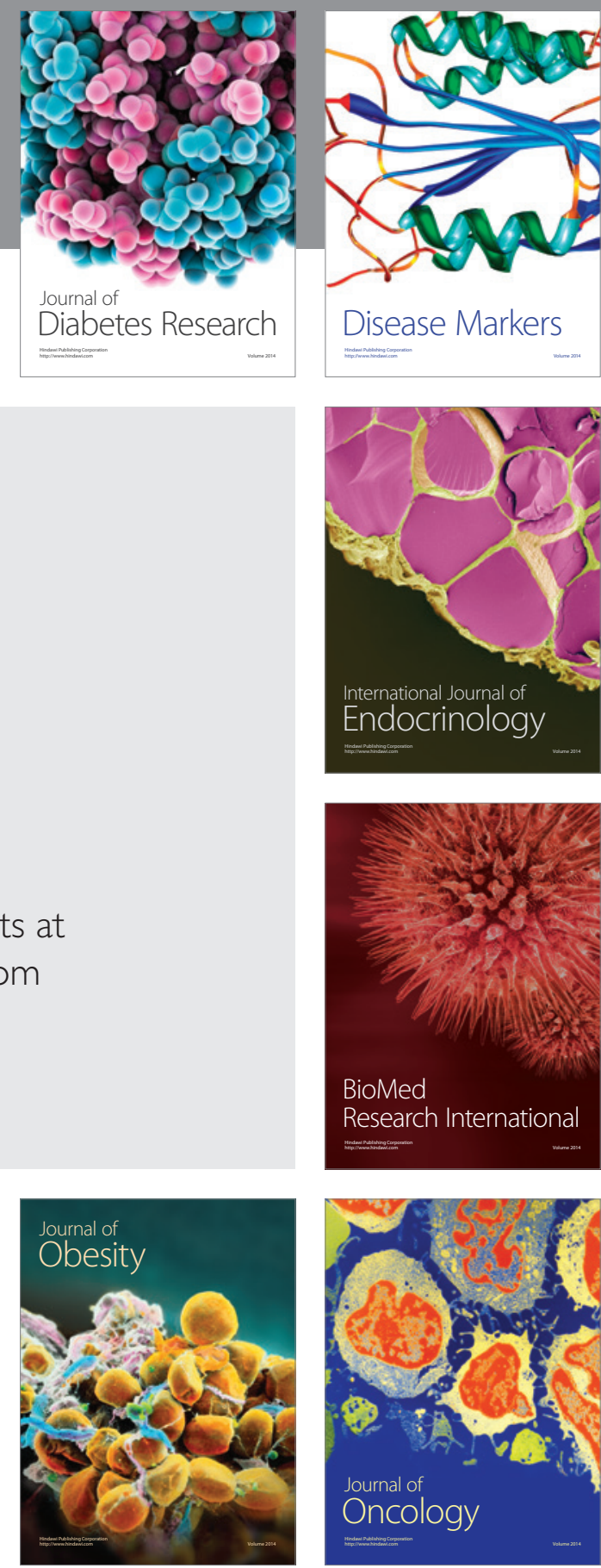

Disease Markers
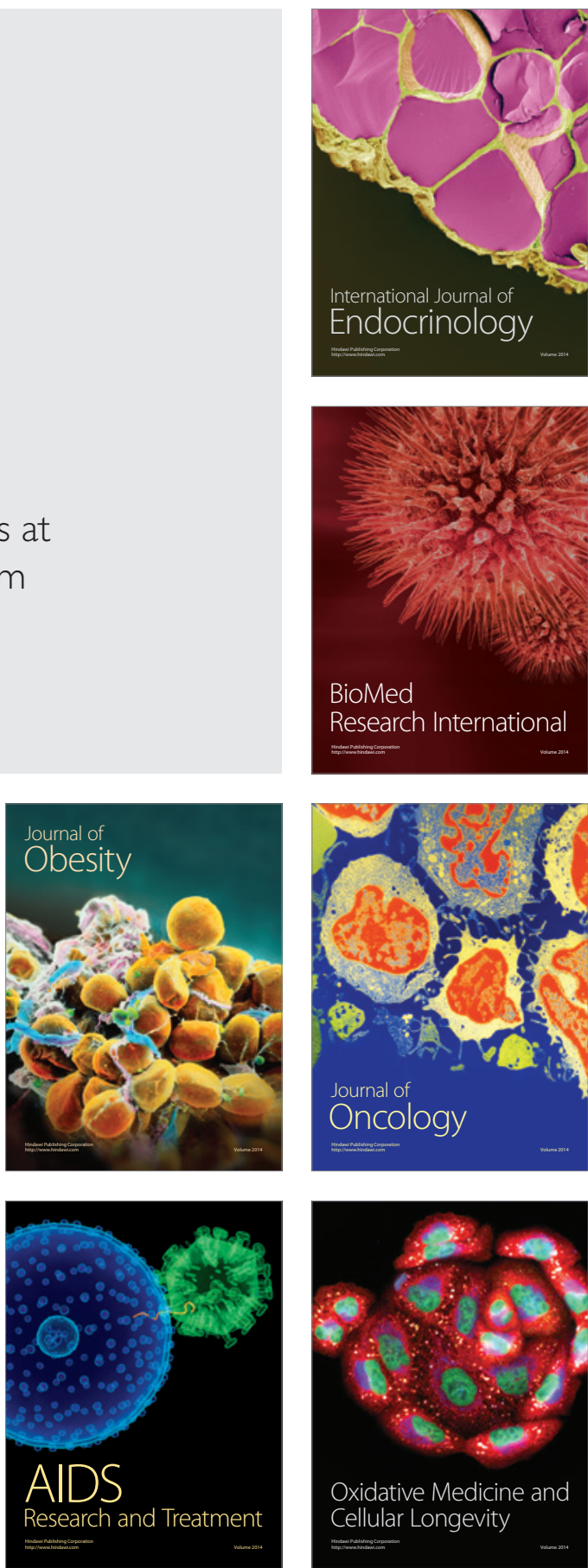